\title{
Pharmacovigilance of Oncology Biosimilars
}

\section{Luis H. Camacho* and Nikhil Pai}

Center for Oncology and Blood Disorders, Houston, TX 77030, USA

"Corresponding author: Luis H. Camacho, MD, MPH, Center for Oncology and Blood Disorders, 6560 Fannin Street. Suite 1224 , Houston, TX 77030 USA, Tel: +1-713-796-1200; Fax: +1-713-795-0735; E-mail: Ihcamacho@cobd.us

Received date: July 07, 2015; Accepted date: July 21, 2015; Published date: July 30, 2015

Copyright: (c) 2015 Camacho LH, et al. This is an open-access article distributed under the terms of the Creative Commons Attribution License, which permits unrestricted use, distribution, and reproduction in any medium, provided the original author and source are credited.

\begin{abstract}
Biologicals are critical in cancer medicine. Four of the top ten biological blockbusters worldwide are oncology drugs used for therapeutic or supportive care. The global market for biologic cancer therapies approximately totalled US $\$ 51.2$ billion in 2014 and is expected to reach US\$ 66.4 billion in 2019 . The patents of most of these top-selling agents will expire by the year 2020 attracting industry to develop biosimilars - agents with high similarity to their biological reference product. Furthermore, manufacturing biosimilars is more cost and time effective than developing their reference products. The cost containments derived from the biosimilars entry to the market are expected to result in considerable societal savings and financial relief to health systems globally. Led by the European Medicines Agency, and the Food and Drug Administration, several other regulatory agencies continue to develop their approval and pharmacovigilance (PV) programs for biosimilars worldwide. Maximizing the safety monitoring and reporting of adverse events (AEs) associated with biosimilars will be critical to rapidly find a place in cancer medicine. Greater efforts to educate patients and healthcare providers to report AEs as well as requesting periodical dissemination and publication of reports by sponsors will be determinant to ensure the post-marketing safety of these agents and gaining the trust of health care providers. This article reviews common and postmarketing AEs associated with the three top cancer biologicals with upcoming expiration patents, their reported AEs during post-marketing surveillance and discusses potential challenges in the PV process of cancer biosimilars.
\end{abstract}

Keywords: Biologic; Biosimilars; Cancer; Oncology; Pharmacovigilance; Postmarketing

\section{Introduction}

Biological agents are critical for cancer therapeutics. Four of the top ten biological blockbusters worldwide are either therapeutic or supportive care oncology agents. Moreover, by year 2020, the patents of these four top cancer biological agents will expire in Europe and the United States [1]. A report by BCC Research estimates that the global market for biologic cancer therapies totaled approximately US $\$ 51.2$ billion in 2014 and is expected to reach US $\$ 66.4$ billion in 2019 . With an estimated annual growth rate of $5.4 \%$ biologicals represent an important financial opportunity for drug manufacturers[2].

Biosimilars are agents with high similarity to their biological reference product. Developing biosimilar agents is less expensive and faster than the process for reference biologicals. A study by the IMS Institute for Healthcare informatics found a median decrease of $35 \%$ in the erythropoietin treatment costs among 21 European countries over the seven year period after erythropoietin biosimilars were marketed [3].

The development and marketing of biosimilars for cancer therapeutics is of particular interest for several reasons: 1) great financial incentive exists for companies to compete for a market share of multiple clinical indications once the patents of the reference products expire, 2) developing these agents at lower costs than their reference biologicals could result in major cost containments and savings for health care globally, 3) less expensive manufacturing and marketing processes will translate in increased access to populations worldwide.
The study endpoints for biosimilars in the US may be different from those used in the reference product's clinical studies if scientifically supported"[4]. Similarly, the EMA states that "the trials guiding principle is to demonstrate similar efficacy and safety compared to the reference medicinal product, not patient benefit per se, which has already been established by the reference medicinal product" [5]. Postmarketing safety monitoring is of critical importance in the development process of biosimilars and manufacturers of biosimilars are required to include a PV program as part of their application in Europe and the United States.

To study the potential challenges faced by PV programs for oncology biosimilars, we reviewed the toxicities outlined by the package inserts of the top three reference cancer biologicals with patent expiration in the next five years and searched for publications describing toxicities observed in their post-marketing experience. Based on our findings, we made preliminary observations to help us identify future needs related to this field.

\section{Cancer Biosimilars}

The study and development of biosimilars is relatively a new discipline. A detailed search on the National Library of Medicine publication records found the first scientific article published under the search term "biosimilars" in 2004[6]. At a growth of 20-30 articles per year, a total of 592 manuscripts were published at the time of preparation of this manuscript. Furthermore, the first article addressing biosimilars in cancer medicine was published in 2008 [7]. To date, only 63 publications discuss manufacturing and regulatory aspects of biosimilars in oncology (Figure 1). These figures suggest that although young, the field involving the development of cancer 
biosimilars has attracted substantial interest in recent years and is likely to continue rapidly expanding.

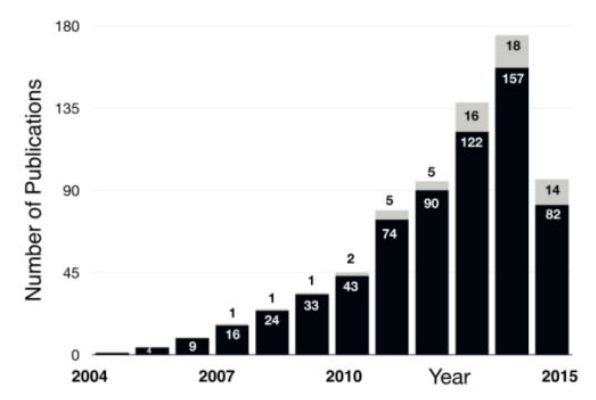

Figure 1: Number of peer reviewed publications listed by the National Library of Medicine (www.pubmed.org) under the term "biosimilars" (black bar) and "biosimilars AND cancer OR oncology" (gray bars) between 2004 (first publication) and June 2015 (at time of submission).

In a survey conducted by the National Comprehensive Cancer Network (NCCN) at their 16th conference, 277 attendees were interviewed to explore how familiar health care providers were with biosimilars. Of the 277 individuals surveyed 129 (46.6\%) were physicians whose response options ranged from extremely familiar with biosimilars to unfamiliar. The authors of the study found that in 2011, almost a decade after the first manuscript on biosimilars was published, $80 \%$ of the physicians surveyed were unfamiliar, slightly familiar, or somewhat familiar with biosimilars. These results are consistent with similar other surveys published over the past 3 years and reflect the slow growth of dissemination of biosimilar knowledge among physicians (Figure 2) [8].

\begin{tabular}{|c|c|c|c|c|c|c|}
\hline Commercial & Active Ingredient & Class & Indication & Company & $\begin{array}{l}\text { Global Sales } \\
\text { (US \$ billion) }\end{array}$ & $\begin{array}{l}\text { Patent Expiration } \\
\text { EU/US }\end{array}$ \\
\hline Humira & adalimumab & TNF Inhibitor & Rheumatology & Abbott/Eisai & 10.7 & Apr 2018/Dec 2016 \\
\hline Remicade & infliximab & TNF Inhibitor & Rheumatology & Merck/Mitsubishi & 8.9 & Aug 2014/Sep 2018 \\
\hline $\begin{array}{l}\text { Rituximab/ } \\
\text { MabThera }\end{array}$ & rituximab & Anti-CD20 & $\begin{array}{l}\text { Rheumatology } \\
\text { Cancer }\end{array}$ & Roche/Biogen-Idec & 8.6 & Nov 2013/Dec 2018 \\
\hline Enbrel & etanercept & TNF inhibitor & Rheumatology & Amgen/Pfizer/Takeda & 8.3 & Feb 2015/Nov 2018 \\
\hline Lantus & insulin glargine & Insulin receptor & Diabetes & Sanofi & 7.8 & $2014 / 2014$ \\
\hline Avastin & bevacizumab & $\begin{array}{l}\text { Anti- } \\
\text { angiogenesis }\end{array}$ & Cancer & Roche & 7.0 & Jan 2022/ Jul 2019 \\
\hline Herceptin & trastuzumab & Anti-HER2 & Cancer & Roche & 6.8 & Jul 2014/ Jun 2019 \\
\hline Neulasta & pegfilgrastim & G-CSF & Neutropenia & Amgen & 4.4 & Aug 2007/ Oct 2015 \\
\hline
\end{tabular}

Table 1: GaBI online - Generics and Biosimilars Initiative. Top 8 cancer biologicals 2013 [www.gabionline.net]. Mol, Belgium: Pro pharma Communications International; [cited Aug 2015]. Available from: www.gabionline.net/Biosimilars/Biosimilars/General/Top-8-blockbusterbiologicals-2013.

\section{Rituximab}

(Rituxan ${ }^{\oplus}$, MabThera $^{\oplus}$, Zytux ${ }^{\oplus}$; Biogen, Cambridge, MA, USA) is a genetically engineered chimeric mouse anti-human monoclonal antibody that targets CD20 on the surface of malignant and normal B lymphocytes. CD20 is a transmembrane protein expressed on all Bcells. The protein is downregulated when B cells differentiate into

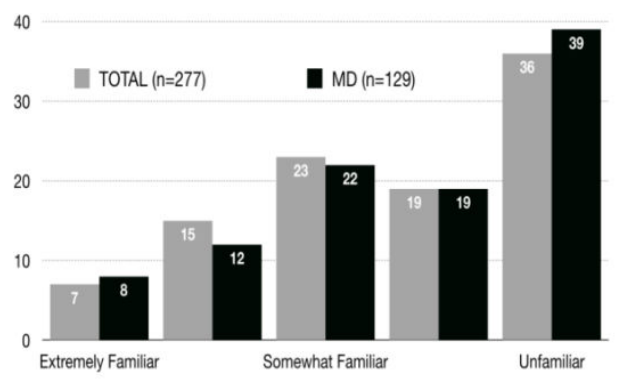

Figure 2: Evaluation of the familiarity with biosimilars among 277 individuals (TOTAL / gray bars) attending a scientific meeting. Of them, 129 were physicians (MD / black bars). Response options included extremely familiar, very familiar, somewhat familiar, slightly familiar, and unfamiliar.

\section{Biologicals in Cancer Medicine}

Three cancer biologicals are among the top eight best-selling biologicals. They include rituximab, trastuzumab, and Bevacizumab (Table 1). Apart from etanercept $\left(\right.$ Enbrel $^{\circledR}$ ) where the US patent has been extended to 2028, the patents on almost all of these biological blockbusters will expire by 2020. Considering these eight biologicals alone, a US $\$ 63$ billion market will open in sales to competition for biosimilars. The biosimilars market earned revenues of approximately US $\$ 172$ million in 2010 and with global sales of biologicals on the rise, these figures will only increase. 
Page 3 of 6

\section{Approved indications}

Rituximab has received FDA approval for 1) certain Non-Hodgkin lymphoma patients, 2) maintenance therapy for untreated follicular CD20 positive B-cell Non-Hodgkin lymphoma; 3) chronic lymphocytic leukaemia (CLL); 4) low-grade or follicular B-cell, CD20 positive Non-Hodgkin lymphoma; 5) diffuse large B-cell, CD20 positive Non-Hodgkin lymphoma; 6) rheumatoid Arthritis (RA) in combination with methotrexate in adult patients with moderately-to severely-active RA who have inadequate response to one or more TNF antagonist therapies; 7) granulomatosis with polyangiitis (GPA); and 8) (Wegener's granulomatosis) and microscopic polyangiitis (MPA) in adult patients in combination with glucocorticoids.

\section{Safety}

Approximately $99 \%$ of patients treated with rituximab experience adverse reactions of different grades of severity and about one-half of these patients experience grade 3 or 4 toxicities. The approval in the United States includes a black box warning about infusion reactions, tumor lysis syndrome, severe mucocutaneous reactions, and progressive multifocal encephalopathy. The most common adverse reactions of Rituxan (occurring $>25 \%$ ) observed in clinical trials of patients with Non-Hodgkin's lymphoma were infusion reactions, fever, lymphopenia, chills, infection, and asthenia [10].

\section{Postmarketing publications}

A Pubmed search with the terms "pharmacovigilance" AND "rituximab" found 17 records; "postmarketing" AND "rituximab" found 18; and "post-marketing" AND "rituximab" found 9. The majority of publications address the incidence of progressive multifocal leukoencephalopathy. While a well-known complication of rituximab therapy, the incidence in different PV databases revealed an incidence between $0.02 \%$ and $0.20 \%$ and in line with the pre-approval clinical trials [11-13]. Investigators have also explored an association with tuberculosis [14], and others described a potential association with melanoma among patients with rheumatoid arthritis and other autoimmune disorders. A total of 69 records associated with rituximab therapy were found among almost 50,000 cases registered in the Spanish PV system. Among the AEs reported, leukocyte disorders, dyspnea, hypotension, and pneumonitis are among the most common and similar to the incidence described in pre-approval trials[15]. Finally, late onset neutropenia has also been reported after rituximab's approval. While the incidence of neutropenia, thrombocytopenia, and anemia were $4.2 \%, 1.7 \%$, and $1.1 \%$ respectively in pre-approval clinical studies, different post-marketing analysis have reported an incidence of neutropenia between 25 and 35\% [16-18].

\section{Bevacizumab}

(Avastin ${ }^{\oplus}$, Genentech, South San Francisco, CA) is a humanized IgG monoclonal antibody produced by DNA technology in Chinese hamster ovary cells[19]. Bevacizumab targets all isoforms of human vascular endothelial factor - (VEGF-A) preventing its binding to the endothelial cell surface receptors VEGFR-1 (Flt-1), and VEGR-2 (KDR/Flk-1). Blockade of these receptor-ligand interactions results in inhibition of angiogenesis and tumor growth. Bevacizumab was first approved by the FDA on February 26, 2004. Patent expiration will be July 2019 in the United States and April 2018 in Europe.

\section{Approved indications}

Bevacizumab has received FDA approval for the following clinical indications: 1) Platinum-resistant recurrent epithelial ovarian, fallopian tube, or primary peritoneal cancer in combination with chemotherapy; 2) Persistent, recurrent, or metastatic cervical cancer in combination with chemotherapy; 3) Metastatic colorectal cancer in combination with Fluoropyrimidine-based chemotherapy for first or second line treatment; 4) Metastatic HER2 negative breast cancer; 5) Metastatic renal cell carcinoma; 6) Second-line treatment of glioblastoma; 7) First-line treatment of non-small cell lung cancer (NSCLC).

\section{Safety}

The approval of Bevacizumab was granted with a black label box warning for gastrointestinal perforation in $3.2 \%$ of all patients, wound healing complications including wound dehiscence, and hemorrhagic events involving different systems including the gastrointestinal, genitourinary tracts, and the central nervous system. The most common adverse reactions observed among patients treated with this agent $(>10 \%)$ and at least twice the control arm rate are epistaxis, headache, hypertension, rhinitis, proteinuria, taste alteration, dry skin, rectal hemorrhage, lacrimation, back pain and exfoliative dermatitis. Furthermore, bevacizumab may exacerbate these reactions when combined with chemotherapeutic agents [20].

\section{Postmarketing publications}

A Pubmed search with the terms "pharmacovigilance" AND "bevacizumab" found 9 records; "postmarketing" AND "bevacizumab" found 11 records, and "post-marketing AND bevacizumab" found 8 records. Eighteen cases of nasal septum perforation have been reported during the post-marketing safety monitoring of Bevacizumab since its original approval in 2004[21]. A review of the French PV database found 351 serious toxicity cases involving 626 AEs. The gastrointestinal tract was most commonly involved (21.9\%). The most frequent AEs included gastrointestinal perforation (4.8\%), thromboembolic events (4.0\%), pulmonary embolism (3.2\%), hypertension $(2.7 \%)$, gastrointestinal hemorrhage (2.7\%), and vascular accidents $(2.6 \%)$. The toxicities reported to the French PV database were similar to those observed during the development of bevacizumab[22]. A review of the same database for drugs associated with induced immune thrombocytopenic purpura also found Bevacizumab in association with thrombocytopenia [23].

\section{Trastuzumab}

(Herceptin $^{\oplus}$, Genentech Inc, South San Francisco, CA) is a recombinant humanized monoclonal antibody directed against an extracellular region of the HER2 protein [24]. The human epidermal growth factor receptor 2 (HER2) is a member of the epidermal growth factor receptor (EGFR) family of receptor tyrosine kinases. Signaling through HER2 ultimately results in cellular proliferation. Overexpression of HER2, with subsequent constitutive kinase activation, is found in approximately one third of human breast and gastric carcinomas [25], and is associated with reduced overall survival. Trastuzumab received FDA approval on September 25, 1998. The EU patent expired in July 2014 and the US patent will expire in June 2019. 
Page 4 of 6

\section{Approved indications}

Trastuzumab has received FDA approved for the following indications: 1) HER2-overexpressing Metastatic Gastric or Gastroesophageal (GE) Junction Adenocarcinoma and 2) HER2overexpressing Breast Cancer.

\section{Safety}

The approval of trastuzumab includes a black box warning for cardiomyopathy especially when administered concomitantly with anthracyclins $(1.4 \%-15 \%)$, infusion reactions and pulmonary toxicities, and embryofetal toxicities[26]. The most common adverse reactions in breast cancer patients receiving trastuzumab in the adjuvant and metastatic settings include fever, nausea, vomiting, infusion reactions, diarrhea, infections, cough, headache, fatigue, dyspnea, rash, neutropenia, anemia, and myalgia. The most common adverse reactions requiring interruption or discontinuation of trastuzumab in clinical trials included congestive heart failure, significant decline in left ventricular cardiac function, severe infusion reactions, and pulmonary toxicity [26].

\section{Postmarketing publications}

A Pubmed search with the terms "pharmacovigilance" AND "trastuzumab" found 3 records; "postmarketing" AND "trastuzumab" found 7 records, and "post-marketing" AND "trastuzumab" found 2 records. The trastuzumab PV monitoring program has served to further identify the characteristics and incidence of congestive heart failure and its association with concomitant anthracyclin therapy [27]. However, toxicities not previously reported have also been observed.

\section{Postmarketing safety monitoring}

According to the World Health Organization, PV is "the science and activities relating to the detection, assessment, understanding and prevention of adverse effects or any other drug-related problem" [28]. $\mathrm{PV}$ is a critical component of marketing programs for approved drugs.

The Prescription Drug User Fee Act (PDUFA) provides authority for the FDA to collect additional resources (fees from industry) and enable the FDA to accelerate its drug evaluation process without compromising the quality of those reviews. On June 12, 2002, the US Congress reauthorized the Prescription Drug User Fee Act (PDUFA III). As a result, the FDA agreed to further implement good PV practices and pharmacoepidemiologic assessment (PV guidance) [29]. After a thorough process and a public workshop in April 2003, an industry guidance document was published [29]. This document serves as a guidance safety signal identification, pharmacoepidemiologic assessment and safety signal interpretation and PV planning and development. Similarly, recognizing PV's safety importance to the public, a new legislation was adopted by the EMA in 2010 to "enhance PV, particularly to support the collection, management and analysis of data, information and knowledge" [30].

A good PV practice must include a well-balanced program that includes safety signal identification, pharmacoepidemiologic assessment and interpretation of signals, and a PV plan development. Case reports by consumers, health care providers, and sponsor officials are important signals of the safety profile of a drug. When a signal is detected, sponsors must record all associated events, gather all data, and publish case series [29]. A rigorous assessment of these events allows sponsors to identify safety signals that warrant further investigation. In this context, it is also important to calculate and understand the difference between the number of reported events and the real incidence of those events in a particular population to avoid bias.

Other mechanisms to investigate safety signals include pharmacoepidemiologic studies, registries, and surveys. The FDA recommends that "a PV plan describes PV efforts above and beyond routine postmarketing spontaneous reporting, and is designed to enhance and expedite the sponsor's acquisition of safety information" [29]. The EMA and FDA applications for biosimilars include a postmarketing safety monitoring plan. There are no critical differences between FDA and EMA recommendations of biosimilar PV. The FDA also states that: "Postmarketing safety monitoring for a proposed product should also have adequate mechanisms in place to differentiate between the adverse events associated with the proposed product and those associated with the reference product, including the identification of adverse events associated with the proposed product that have not been previously associated with the reference product". This important aspect of biosimilar PV addresses the potential incidence of serious and rare $(<1 \%)$ events. Under such circumstances, the FDA proposes that: "Rare, but potentially serious, safety risks (e.g., immunogenicity) may not be detected during preapproval clinical testing because the size of the population exposed likely will not be large enough to assess rare events. In particular cases, such risks may need to be evaluated through postmarketing surveillance or studies" [4].

\section{Immunogenicity}

The induction of immune reactions against an agent may be driven by the agent itself, the manufacturing process, or host's factors. Immunogenicity may result in hypersensitivity, anaphylaxis, and loss of efficacy. Several factors surrounding manufacturing and patient characteristics may result in immunogenicity of a drug [31,32]. Postmarketing studies aimed at studying the immunogenicity of biosimilars are also very important in ensuring the safety of the population.

Among the best-known examples of product immunogenicity resulted from the substitution of polysorbate 80 and glycin for albumin as a stabilizer in the Eprex formulation of Epoietin alfa. This modification resulted in the development of a neutralizing antibody mediated pure red cell aplasia [31]. Approximately 175 cases were identified between 1998 and 2004 in Europe. These and subsequent events associated with erythropoietin biosimilars raised awareness of the importance of immunogenicity in the development and production of biological agents with the need to rapidly identify deficiencies and correcting them.

PV programs following safety signals for immunogenicity will be especially necessary in the development of cancer biosimilars were efficacy may be impaired in addition to potential serious allergic reactions.

\section{Pharmacovigilance of Cancer Biosimilars: Considerations}

Cancer patients experience a multitude of signs and symptoms associated with their condition and/or the treatments they receive. Collectively, these symptoms have been termed symptom burden, and may be the source of significant distress and function impairment [33]. 
Page 5 of 6

Evaluation of postmarketing safety monitoring of biosimilars may pose special challenge in cancer patients due to several characteristics of their disease process:

\section{Neoplastic processes may be associated with substantial symptom burden associated}

Cancer patients receiving biological therapies are often debilitated by their condition and/or treatment. Unfortunately, patients and health care providers may assume their AEs are part of the disease process, and under-report. An example of the high incidence of symptom burden experienced by patients with advanced cancer is reflected in a Phase III clinical trial that led to the approval of ipilimumab - developed for the treatment of patients with unresectable melanoma, where 676 patients with metastatic melanoma were randomized to receive the monoclonal antibody directed against CTLA4 - a cytoplasmic receptor expressed by T-cells shortly after their activation (131), a peptide vaccine (132), or both agents (380) [34]. In this study, the incidence of adverse events of all intensities was observed in $97 \%$ of patients randomized to receive the peptide vaccine and in $96.9 \%$ of the patients who received the antibody alone. While not exactly a placebo, peptide vaccines are among the least toxic biologicals in cancer medicine, and hence, not expected to have a toxic profile. Roughly, a third of the patients in each arm of this trial experienced nausea and fatigue, whereas one fifth of the patients in the vaccine arm experienced diarrhea, constipation, vomiting, decreased appetite, pyrexia, dyspnea, or anemia, leading one to believe these adverse events were very likely, secondary to their disease process itself. Of note, the vaccine had previously proven safe in the Phase I clinical trial conducted by the surgical branch group at the National Cancer Institute in 10 patients with similar characteristics. Such studies are traditionally designed to explore the toxicities and tolerability of an agent, demonstrated no concerning AEs [35]. Finally, biologicals are often administered beyond second line therapies when patients are further debilitated and a greater incidence of confounding symptoms present [36].

\section{The approval process for reference biologicals requires larger clinical trials than the process for biosimilars}

Survival is generally a required endpoint in oncology clinical trials and demonstrating statistically significant improvements requires large numbers of patients followed over long periods of time. In contrast, the approval of biosimilars requires endpoints that can assess clinically meaningful differences between the proposed product and the reference product in comparative clinical studies. These studies are much smaller and their follow up substantially shorter. It is more difficult to detect unusual AEs in studies with limited numbers of patients. In addition to following and reporting uncommon AEs, close monitoring for long-term toxicities is critical. A higher risk for melanoma among patients treated with rituximab has also been identified and reported during its postmarketing period [37]. However, it is important to note that these reports included patients who received several immune-suppressive agents to treat autoimmune conditions prior and after rituximab therapy and hence, a causality effect is difficult to establish with accuracy.

\section{Communication of label warnings to patients and health care providers is a slow process}

The package insert of a medication is a brief document that includes detailed drug information compiled and distributed by the drug manufacturer after its review and approval by the FDA. The purpose of the package insert is to provide complete and unbiased prescribing and safety information to health care professionals [38]. Package inserts include all AEs reported by the sponsor on the studies submitted in the application dossier. However, it takes on average 7 to 10 years for a "black box" warning to be added to the package insert after a medication approval [39]. This long delay may pose safety concerns.

\section{The publication rate of postmarketing AEs is exceedingly low}

Despite the FDA recommendations to sponsors and healthcare providers to periodically publish case reports and case series, our review of the postmarketing reports in peer review journals for three top cancer biologicals revealed between 2 and 18 publications in over 10 years of marketing.

\section{Summary}

Biological agents are of critical importance in oncology. The patent expiration of several top cancer biologicals with global sales greater than US\$ 20 billion will open a large market share for drug developing companies worldwide. The entry of biosimilars in the market of cancer therapeutics is likely to decrease the costs of healthcare and expand the access to biologicals. Early experience in Europe with erythropoietin biosimilars suggests that on average, the costs of treatment could be reduced by $35 \%$.

It is highly expected that the associated AEs be very similar based on thorough evaluations of the pharmacology, production, safety, and immunogenicity data and processes presented for approval of biosimilars in comparison with their reference product. However, reference products are approved after a careful analysis of thousands of patients enrolled in clinical trials and unlike reference products, biosimilars are approved on the basis of similar safety and efficacy through much smaller studies. While close follow up of thousands of patients would be desirable, a large number of patients monitored after marketing of the product will allow the identification of uncommon events. While PV databases are robust and include large numbers of patients, the communication and dissemination of reported AEs is usually made through regular FDA communications, which may not reach healthcare providers in a timely fashion.

Finally, similar to others, we found a deficit of scientific publications reporting on the safety monitoring of biologicals [11]. A Pubmed search for each of the three top cancer biologicals with upcoming patient expiration demonstrated only between 2 and 18 publications since their FDA approval over one decade ago. While mandatory to include a PV program in the biosimilar application to the FDA and the EMA, the development of policies educating and encouraging health care providers and patients to report AEs would further improve the safety monitoring of biosimilars and increasing the trust among patients and providers. Beyond FDA encouragement, mandatory requirements should be in place for sponsors to periodically publish the AEs reported after marketing of an agent. 
Page 6 of 6

Ultimately, the responsibility for identifying safety signals should be shared by healthcare providers, sponsors, and the FDA.

\section{References:}

1. GaBI (2014) Top 8 blockbuster biologicals 2013. In Generics and Biosimilars Initiative.

2. BCC Research (2015) Biological Therapies for Cancer: Technologies and Global Markets.

3. Institute for Healthcare Informatics (2014) Assessing Biosimilar Uptake and Competition in European Markets.

4. FDA (2015) Scientific Considerations in Demonstrating Biosimilarity to a Reference Product.

5. European Medicines Agency (2012) Guideline on similar biological medicinal products containing monoclonal antibodies - non-clinical and clinical issues.

6. Schellekens H (2004) How similar do 'biosimilars' need to be? Nat Biotechnol 22: 1357-1359.

7. Mellstedt H, Niederwieser D, Ludwig H (2008) The challenge of biosimilars. Ann Oncol 19: 411-419.

8. Zelenetz AD, Ahmed I, Braud EL, Cross JD, Davenport-Ennis N, et al. (2011) NCCN Biosimilars White Paper: regulatory, scientific, and patient safety perspectives. Journal of the National Comprehensive Cancer Network 4: S1-S22.

9. Smith MR (2003) Rituximab (monoclonal anti-CD20 antibody): mechanisms of action and resistance. Oncogene 22: 7359-7368.

10. Genentech. (2014) Rituximab: Package Insert - Prescribing Information.

11. Bennett CL (2011) Pharmacovigilance and PML in the oncology setting. Cleve Clin J Med 78 Suppl 2: S13-17.

12. Bosch X, Saiz A, Ramos-Casals M; BIOGEAS Study Group (2011) Monoclonal antibody therapy-associated neurological disorders. Nat Rev Neurol 7: 165-172.

13. Allison M (2010) PML problems loom for Rituxan. Nat Biotechnol 28: 105-106.

14. Cantini F, Niccoli L, Goletti D (2014) Tuberculosis risk in patients treated with non-anti-tumor necrosis factor- $\hat{I} \pm$ (TNF-Î \pm ) targeted biologics and recently licensed TNF-Ît inhibitors: data from clinical trials and national registries. J Rheumatol Suppl 91: 56-64.

15. González V, Salgueiro E, Jimeno FJ, Hidalgo A, Rubio T, et al. (2008) Post-marketing safety of antineoplasic monoclonal antibodies: rituximab and trastuzumab. Pharmacoepidemiol Drug Saf 17: 714-721.

16. Dunleavy K, Tay K, Wilson WH (2010) Rituximab-associated neutropenia. Semin Hematol 47: 180-186.

17. Nitta E, Izutsu K, Sato T, Ota Y, Takeuchi K, et al. (2007) A high incidence of late-onset neutropenia following rituximab-containing chemotherapy as a primary treatment of CD20-positive B-cell lymphoma: a single-institution study. Annals of oncology 18: 364-369.

18. Cattaneo C, Spedini P, Casari S, Re A, Tucci A et al. (2006) Delayedonset peripheral blood cytopenia after rituximab: frequency and risk factor assessment in a consecutive series of 77 treatments. Leukemia \& lymphoma 47: 1013-1017.

19. Keating GM (2014) Bevacizumab: a review of its use in advanced cancer. Drugs 74: 1891-1925.

20. Genentech. (2014) Bevacizumab: Package insert - prescribing information.

21. Ramiscal JA, Jatoi A (2011) Bevacizumab-induced nasal septal perforation: incidence of symptomatic, confirmed event(s) in colorectal cancer patients. Acta Oncol 50: 578-581.

This article was originally published in a special issue, entitled: "Biosimilars Pharmacovigilance and Risk Management", Edited by Eugenia

Yiannakopoulou, Faculty of Health and Caring Professions, Technological Educational Institute of Athens, Greece
22. Taugourdeau-Raymond S, Rouby F, Default A, Jean-Pastor MJ, French Network of Pharmacovigilance C (2012) Bevacizumab-induced serious side-effects: a review of the French pharmacovigilance database. European journal of clinical pharmacology 68: 1103-1107.

23. Moulis G, Sommet A, Sailler L, Lapeyre-Mestre M, Montastruc JL; French Association Of Regional Pharmacovigilance Centers (2012) Drug-induced immune thrombocytopenia: a descriptive survey in the French PharmacoVigilance database. Platelets 23: 490-494.

24. Carter P, Presta L, Gorman CM, Ridgway JB, Henner D, et al. (1992) Humanization of an anti-p185HER2 antibody for human cancer therapy. Proc Natl Acad Sci USA 89: 4285-4289.

25. Gravalos C, Jimeno A (2008) HER2 in gastric cancer: a new prognostic factor and a novel therapeutic target. Ann Oncol 19: 1523-1529.

26. Genentech. (2015) Trastuzumab: Package insert and prescribing information

27. Bowles EJ, Wellman R, Feigelson HS, Onitilo AA, Freedman AN, et al. (2012) Risk of heart failure in breast cancer patients after anthracycline and trastuzumab treatment: a retrospective cohort study. J Natl Cancer Inst 104: 1293-1305.

28. WHO (2015) Pharmacovigilance of Essential Medicines and Health Products.

29. FDA (2005) Good Pharmacovigilance Practices and Pharmacoepidemiologic Assessment: Guidance for Industry.

30. Directive 2010/84/Eu of the European Parliament and of the Council (2010) Official Journal of the European Union.

31. Bennett CL, Luminari S, Nissenson AR, Tallman MS, Klinge SA, et al. (2004) Pure red-cell aplasia and epoetin therapy. N Engl J Med 351: 1403-1408.

32. Evens AM, Bennett CL, Luminari S (2005) Epoetin-induced pure red-cell aplasia (PRCA): preliminary results from the research on adverse drug events and reports (RADAR) group. Best Pract Res Clin Haematol 18: 481-489.

33. Cleeland CS (2007) Symptom burden: multiple symptoms and their impact as patient-reported outcomes. J Natl Cancer Inst Monogr 16-21.

34. Hodi FS, O'Day SJ, McDermott DF, Weber RW, Sosman JA, et al. (2010) Improved survival with ipilimumab in patients with metastatic melanoma. N Engl J Med 363: 711-723.

35. Panelli MC, Wunderlich J, Jeffries J, Wang E, Mixon A, et al. (2000) Phase 1 study in patients with metastatic melanoma of immunization with dendritic cells presenting epitopes derived from the melanomaassociated antigens MART-1 and gp100. J Immunother 23: 487-498.

36. Giezen TJ, Straus SM (2012) Pharmacovigilance of Biosimilars: challenges and possible solutions. Generics and Biosimilars Initiative Journal (GaBI Journal) 1: 118-119.

37. Peuvrel L, Chiffoleau A, Quéreux G, Brocard A, Saint-Jean M, et al (2013) Melanoma and rituximab: an incidental association? Dermatology 226: 274-278.

38. Watson KT, Barash PG (2009) The new Food and Drug Administration drug package insert: implications for patient safety and clinical care. Anesth Analg 108: 211-218.

39. Lasser KE, Allen PD, Woolhandler SJ, Himmelstein DU, Wolfe SM, et al. (2002) Timing of new black box warnings and withdrawals for prescription medications. JAMA 287: 2215-2220. 